\title{
Consumer Health Informatics: The Application of ICT in Improving Patient-Provider Partnership for a Better Health Care
}

\author{
Benjamin Abaidoo', Benjamin Teye Larweh ${ }^{2}$ \\ ${ }^{1}$ University of Ghana Medical School Korle Bu, Department of Surgery, Eye Unit \\ ${ }^{2}$ Ghana Cocoa Board, Public Affairs Department.
}

\begin{abstract}
Background: There is a growing interest concerning the potential of ICT solutions that are customized to consumers. This emerging discipline referred to as consumer health informatics $(\mathrm{CHI})$ plays a major role in providing information to patients and the public, and facilitates the promotion of self-management. The concept of $\mathrm{CHI}$ has emerged out of the desire of most patients to shoulder responsibilities regarding their health and a growing desire of health practitioners to fully appreciate the potential of the patient.

Aim: To describe the role of ICT in improving the patient-provider partnership in consumer health informatics.

Methods: Systematic reviewing of literature, identification of reference sources and formulation of search strategies and manual search regarding the significance of developed $\mathrm{CHI}$ applications in healthcare delivery.

Results: New consumer health IT applications have been developed to be used on a variety of different platforms, including the Web, messaging systems, PDAs, and cell phones. These applications assists patients with self-management through reminders and prompts, delivery of real-time data on a patient's health condition to patients and providers, web-based communication and personal electronic health information.

Conclusion: New tools are being developed for the purposes of providing information to patients and the public which has enhanced decision making in health matters and an avenue for clinicians and consumers to exchange health information for personal and public use. This calls for corroboration among healthcare organizations, governments and the ICT industry to develop new research and IT innovations which are tailored to the health needs of the consumer.
\end{abstract}

Keywords: Consumer health informatics, information communication Technology, patient, provider, clients and internet.

Correspondence: benjamin_abaidoo@yahoo.com

DOI: 10.5210/ojphi.v6i2.4903

Copyright @2014 the author(s)

This is an Open Access article. Authors own copyright of their articles appearing in the Online Journal of Public Health Informatics. Readers may copy articles without permission of the copyright owner(s), as long as the author and OJPHI are acknowledged in the copy and the copy is used for educational, not-for-profit purposes.

\section{Introduction}

There is a growing awareness concerning the potential of information and communication technology and e-health solutions that are modified to meet the health needs of the consumer of 
healthcare services. This emerging field is referred to as consumer health informatics (CHI). Consumer Health Informatics (CHI) plays a major role in providing information to patients and the public, which facilities the promotion of self-care, enabling informed decision-making, promoting healthy behaviors and peer information exchange.

Eysenbach defines CHI as a medical informatics that analyzes consumer's needs for information, studies and implements methods of making information accessible to consumers, and models and integrates consumer's preferences into medical information systems [1].

The American Medical Informatics Association (AMIA) identifies CHI as a subspecialty in medical informatics which studies from a patient/consumer perspective the use of electronic information and communication to improve medical outcomes and the healthcare decisionmaking process [2].

The advent of consumer health related web sites, online support groups and electronic patientcentered communications, presents new challenges for clinical practice. Healthcare givers have an important task of helping their clients and the public locate, assess, and interpret health information [3].

This study adopts the definition of CHI by Gibbons MC. et al [4] who defines CHI as any electronic tool, technology, or system: (a) primarily designed to interact with health information consumers (anyone who seeks or uses healthcare information for non-professional work); (b) that interacts directly with the consumer who provides personalized health information to the to the CHI system and receives personalizes health information from the tool or system and (c) in which the data, information, recommendations or other benefits provided to the consumer may be used with a healthcare professional, but is not dependent on a healthcare professional.

This study describes the consumer of healthcare as any individual who assesses healthcare services (both patients and individuals who are not ill) or information for his/her personal health needs and the provider as the healthcare professional or facility providing health needs to the consumer.

The desire of most clients (patients) to assume more responsibility for their health and the growing desire of health practitioners to fully appreciate the potential of the patient and their families correspond with the emergence of interactive information technology solutions available to clients.

These developments are further fueled by cost-saving pressures in health systems and the hope that giving consumers more information has the potential of increasing quality of care and save costs by fully realizing the self-help potential of the patient and their family.

There is an increasing pressure on healthcare consumers to actively participate in health care resulting in a thirst for health information. Further, there is also an increasing need for health information technologies to assist patients in participating in their own health care [5].

Meke et al [6] surveyed health services providers in Namibia to find out how they use ICTs in the delivery of healthcare. Twenty-one (21) health workers completed a set of structured questionnaire in two regions of Namibia (one urban, one rural). ICTs were seen as very important tool for medical services $(100.00 \%)$. Ninety-one percent $(91.00 \%)$ of health service providers viewed ICT as helping them interact with colleagues. The telephone was the most commonly used ICT tool, which was used in the admission of patients (36.00\%). Personal Computer was the next most commonly ICT (23\%). 
Consumers are now ready to become partners in their own healthcare. A new breed of empowered, computer-literature consumers is slowly redefining the physician-patient relationship [7].

One of the most radical steps towards consumer empowerment is the creation of electronic health records accessible via the Internet. This will enable consumers to practice online-doctoring as in the case of e-commerce and e-banking.

A recent study by the Pew Internet survey revealed that $72 \%$ of Internet users in the USA say they looked online for health information. In this study, the internet became the second most trusted source of information after a personal doctor. Most consumers visit Google before going to esteemed sources for health information and most don't check the origin of the information obtained [5].

Tele-health has great potential of advancing access to quality healthcare. However, its adoption in routine healthcare has been slow and consequently, the lack of clarity about the value of telehealth implementation is a concern for the slow adoption [8].

Lack of face-to-face meetings or privacy did not appear to be a problem for consumers in their use of ICT in communicating with providers [9].

Consumer health informatics developments offer further opportunities and an increasing potential to grant consumers access to relevant information. Integration of personal data with explanatory information may allow elderly consumers access to their electronic medication lists via the internet. Such systems may be integrated with drug information [10].

This study therefore describes the role of ICT in improving the patient-provider partnership in consumer health informatics by looking at current trends and future directions in consumer health informatics, examining issues surrounding consumers' use of health information technology and discussing current applications of ICT in consumer health informatics.

\section{Methods}

Systematic reviewing and blending of available literature and evidence from 1999 to 2013 regarding the impact of developed $\mathrm{CHI}$ tools on healthcare delivery. Searching for literature involved identifying reference sources and formulating a search strategy for each source.

For the searching of electronic databases, medical subject heading (Mesh) terms were used. To identify articles that are potentially relevant to the topic and the objectives, terms relevant to the definition of CHI applications were used including key word search such as consumer, ICT use, patient-provider partnership and informatics. Eligible studies were also reviewed by reviewing the references in pertinent reviews.

Data sources included searched literature of peer reviewed literature databases and grey literature databases as well as hand searching from the MEDLINE, Cochrane Library, Nursing and Allied Health Literature (CINAHL) databases and Google scholar.

\section{Results}

Findings reveal that information technology and consumer health informatics is becoming an important aspect of modern public health practice. 
Currently, existing systems designed for health professionals are being adapted to be used at home by patients. New consumer health IT applications are being developed to be used on different platforms such as the internet, messaging systems, PDAs, and cell phones.

Low-cost communication systems (Skype, handled radios) have been developed to assist patients in communicating with physicians and obtaining vital information pertaining to their health care.

Smartphone applications, Self-Management Systems, Electronic Personal Health Records, Patient Portals, Peer Interaction Systems, Telemedicine and eLearning, mobile health are all ICT tools which facilitates the delivery of quality healthcare to consumers.

E-mail and text-messaging are all vital ICT tools developed to assist the patient and the provider in the quest for good health and quality health care delivery.

\section{Discussion}

Information technology and consumerism are synergistic forces that promote an information age healthcare system in which consumers are able to apply ICT in self-care management and accessing of health information thereby utilizing healthcare resources more efficiently.

ICT applications have now become a conduit in channeling health information to consumers. There are softwares designed to assist consumers in making informed decisions with care givers tailoring interventions appropriately.

Several ICT tools are being developed to enhance quality health information on the internet and also to facilitate the education of consumers.

Consumer Health Informatics tools and their use can benefit both patients and providers [11]. These applications have various purposes including assisting with self-management through reminders and educational prompts, delivering real-time data on a patient's health condition to both patients and caregivers and storing personal health information in an easily accessible format.

One example of the potential benefits of these kinds of applications is illustrated by the use of messaging capabilities available in certain consumer health IT applications that enable timely communications between patients and their providers [12].

Moreover, consumer health IT applications that allow gathering and integrating data from various health care sources can serve as a comprehensive resource for patients and their providers. In addition to convenience, consumer health IT applications are very significant in emergency situations in the provision of critical health information to medical staff.

\section{Applications of Smart phones}

Smart phones adoption has paved way for the retrieval of a wealth of health information quickly and efficiently. Users are able to accessed information through the Internet regularly through their smart phones, including materials relating to health. Users apply smart phones in looking for health information before deciding to consult a doctor. They use it in searching for healthy recipes to cook at home. Users expressed interest in apps that gave information and advice that they could access on the go [13]. 


\section{Self-Management Systems}

This includes systems with different combinations of functionalities utilizing multiple platforms with some providing effective and real-time response to information about the current status of the user. Some of them allow for monitoring and transmission of information, such as blood pressure or blood glucose. Depending on system design, feedback to a patient regarding his/her health status can be received from the system directly or from the provider who receives information from the system.

A transfer of care activities from the healthcare providers to the patients could be achieved by equipping patients with adequate tailored tools (in particular, dedicated for self-management). In this way, patients become real partners in the care process, and healthcare providers can give further attention to the treatment of more serious health conditions [13].

\section{Electronic Personal Health Records and Patients Portals}

Electronic personal health records (PHRs) are defined as an electronic record of health-related information concerning an individual that conforms to nationally recognized interoperability standards and that can be drawn from multiple sources while being managed, shared and controlled by the individual [14].

An electronic PHR can exist as a stand-alone application that allows information to be exported to or imported from other sources or applications or as a tethered application that is linked to a specific health care organizations information system. Tethered PHRs, also referred to as patient portals, typically allow patients to view, but not modify, data from the providers electronic health record (HER). Relevant information that is often retained in a PHR includes personal identifiers, contact information, health provider information, problem list, medication history [15].

Some applications such as the Electronic Health Records also allow patients to communicate electronically with their providers.

\section{Peer Interaction Systems}

The internet has become a very resourceful avenue for clinicians and clients as well in problem solving and emotional support about their health concerns. The internet is not a replacement for doctors, but they can help you exchange information about treatment options and experiences, disabling conditions, care facilities, dealing with complications, current research and many other topics.

Peer interaction can take the form of stand-alone applications or can sometimes be a part of multi-component applications. These applications can increase the perceived peer support and improve personal and social outcomes [13].

\section{Telemedicine and eLearning}

Telemedicine is the use of medical information exchanged from one site to another via electronic communications to improve a patient's clinical health status [16]. Telemedicine helps to improve healthcare services, promote healthy lifestyles and education, where the patients or clients are geographically separated. Telemedicine facilities for clinical consultation include patient assessment, diagnosis and treatment, continuing professional education, health promotion and healthcare management. Its application is very significant in rural healthcare delivery, inmate 
correction facilities, schools, industrial health services, disaster relief organizations, mobile health facilities among others.

\section{Low-cost communication systems}

These include Skype and handheld radios for improved medical communication among health practitioners and their clients.

\section{Health applications web sites}

These web sites can help you track information, analyze it and even save it in a form you can share with your doctor.

\section{Mobile health}

Mobile health applications can help one to collect, analyze and use the same information. Some mobile health applications even the sensors in ones phone to enrich the information collected such as how far one climbed or how fast one ran. Other mobile health applications help you keep track of critical information such as your medication schedule and can provide alerts to keep you on track.

In a recent World Health Organization Global Health Survey, 60\% of high-income countries and $30 \%$ of low and middle-income countries admitted using SMS messages and other mobile health communications tools for improving treatment compliance. The related programs in low and middle-income countries address a variety of priority health concerns, including H1N1 influenza virus infection, HIV infection, vaccination, reproductive health and management of chronic illness $[17,18]$.

\section{E-mail and text messages}

E-mail and text messages are very useful in reminding patients about their appointments and other important information which may be needful in managing a chronic illness.

Literature reviews indicate that SMS messages and other tools for communicating with patients between medical visits can improve health behaviors and physiological outcomes [19,20]. A review of seven interventional studies and four randomized trials revealed text messaging as a significant application for improving adherence rates [19].

Studies on the effectiveness of SMS messages for health promotion have also shown improvements in the outcomes of care [21,22].

In a trial of smoking cessation involving 5800 participants, the percentage of participants who had quit smoking (verified biochemically) had more than double 6 months after a txt2stop intervention [22]

\section{Conclusion:}

Information technology and consumer health informatics are becoming an integral part of modern concepts of public health and national health care policies communication is far from being realized. The Internet has both the consumer who want to know the status of their health 
and the providers whose responsibility is focused on providing better information for a better healthcare and decision making.

As more interactive, consumer-facing applications appear patients are now playing a more active role both in their own health management and in the shaping of services. The shift if responsibility and power from institution to individual has huge implications for healthcare systems worldwide.

A key trend is towards integration of services, technologies, media, data, knowledge and communities. Changes in the organization and funding of care will both influence and be influenced by patient-centered innovations.

Application of ICT in CHI can provide information to patients and the public, promote self-care, enable informed decision-making, promote healthy behaviors and promote peer information exchange and social support. With ICT resources, consumers will feel more confident and empowered with increased knowledge and improved health status.

New consumer health information technology (health IT) applications are being developed that allow patients to manage, share and control their health information electronically and to assume a more active role in the management of their health. Quality, research methodology and accessibility must all be increased to ensure that $\mathrm{CHI}$ achieves its potential to improve the healthcare system.

Further, there is the need for corroboration among healthcare organizations, governments and the ICT industry to develop new research and IT innovations which are tailored to the health needs of the consumer.

\section{Acknowledgment}

We are very grateful for the support accorded us by Mrs. Janet Esi Abaidoo and Ms. Benedicta Kwagyir Entsie.

\section{Conflicts of interest}

None.

\section{Funding Sources}

None.

\section{References:}

1. Eysenbach G. 2000. Consumer Health Informatics. BMJ. 320, 1713-16. PubMed http://dx.doi.org/10.1136/bmj.320.7251.1713

2. American Medical Information Association Consumer Health Informatics Working Group. Available at: http://www.amia.org/programs/working-group/programs/consumer-healthinformatics.

3. Houston, T. \& Ehrenberger, H.. The Potential of Consumer Health Informatics. Seminars in Oncology Nursing.17 (1): 41:47. 
4. Gibbons MC, Wilson RF, Samal L, Lehman CU, Dickersin K, et al. Impact of consumer health informatics applications (Prepared by Johns Hopkins Evidence Based Practice Center Under contract No. HHSA 290-2007-10061-I), editor. Evid Rep Technol Assess (Full.Rep.) 09(10)-E019 [188], 1-546. Ref Type: Report. Retrieved on 8/5/2013.

5. Pew internet. (Retrieved on 10/5/2013).

6. Meke I. Shivute, Blessing M. Maumbe, Vesper T. Owei. 2008. The use of information and communication technology for health service delivery in Namibia: perspectives of the health service providers. $J$ Telemed Telecare. 14(6), 285-89. $\quad \underline{\text { PubMed }}$ http://dx.doi.org/10.1258/jtt.2008.071204

7. Ball M. J, Lillis J. E-health: transforming the physician/patient relationship. Int J Med inform. Apr: 61 (1):1 - 10.

8. Cusack CM. 2008. The value proposition in the widespread use of tele-health. J Telemed Telecare. 14(4), 167-8. PubMed http://dx.doi.org/10.1258/jtt.2007.007043

9. Kerstin M., Akesson, Britt-Inger Saveman, Gunilla Nilsson. International Health Care Consumers experiences of information communication technology - a summary of literature. Journal of Medical Informatics Volume 76, Issue 9, Pages 633-645.

10. Rind DM, Kim JH, Sturges EA. 1999. Connecting patients to their medication records. Proc AMIA Symp. (1-2), 1147.

11. Jimison H, Gorman P. woods. Barriers and Drivers of Health Information Technology Use for the Elderly, Chronically III, and Underserved. Evidence Report/Technology Assessment No. 175 (Prepared by the Oregon Evidence-based Practice Center under Contract No. 29002-0024).

12. Tang PC, Lansky D.. The missing link: bridging the patient-provider health information gap. Health Aff (Millwood) 2005 Sept - Oct: 24 (5): 1290-5. Retrieved on 10/5/2013).

13. Dennison L, Morrison L, Conway G, Yardley L. 2013. Opportunities and Challenges for Smartphone Applications in Supporting Health Behaviour Change: Quantitative Study. $J$ Med Internet Res. 15(4). e86. PubMed http://dx.doi.org/10.2196/jmir.2583

14. The National Alliance for Health Information Technology. Defining Key Health Information Technology Terms.

15. HL7 electronic Health Records Workgroup. PHR-System Functional Model, Release 1 DSTU, November 2007.

16. American Telemedicine Association. Available at: http://www.americantelemed.org/learn.

17. mHealth. New horizons for health through mobile technologies. Geneva: World Health Organization; 2011.

18. Déglise C, Suggs LS, Odematt P.. Short message service (SMS) applications for disease prevention in developing countries. J Med Internet Res 2012; 14: e3-doi: 10.2196/jmir.1823 pmid: 22262730.

19. Wei J, Hollin I, Kachnowski S.. A review of the use of mobile phone text messaging in clinical and healthy behaviour interventions. J Telemed Telecare 2011; 17: 41-8 doi:10.1258/jtt.2010.100322 pmid: 21097565. 
20. Fjeldsoe BS, Marshall AL, Miller YD.. Behaviour change interventions delivered by Mobile telephone short-message service. Am J Prev Med 2009; 36: 165-73. doi: 10.1016/j.amepre.2008.09.040 pmid: 19135907.

21. Haug S, Meyer C, Schorr G, Bauer S, John U. Continuous individual support of smoking cessation using text messaging: a pilot experimental study. Nicotine Tob Res 2009:11:91523doi:10.1093/ntr/ntp084pmid:19542517. http://www.ahrq.gov/clinic/tp/chiapptp.htm. (Retrieved on 8/5/2013).

22. Free C, Knight R, Robertson S, Whittaker R, Edwards P, Zhou W.. Smoking cessation support delivered via mobile phone text messaging (txt2stop): a single-blind, randomized trial, Lancet 2011; 378: 49-55. doi: 10.1016/S0140-6736(11)60701-0 pmid: 21722952. 